University of New Hampshire

University of New Hampshire Scholars' Repository

Space Science Center

Institute for the Study of Earth, Oceans, and

Space (EOS)

2001

\title{
SONTRAC-a scintillating plastic fiber tracking detector for neutron and proton imaging spectroscopy
}

James M. Ryan

University of New Hampshire, James.Ryan@unh.edu

John R. Macri

University of New Hampshire - Main Campus, John.Macri@unh.edu

Mark L. McConnell

University of New Hampshire - Main Campus, mark.mcconnell@unh.edu

R S. Miller

University of New Hampshire - Main Campus

Follow this and additional works at: https://scholars.unh.edu/ssc

Part of the Astrophysics and Astronomy Commons

\section{Recommended Citation}

SONTRAC-a scintillating plastic fiber tracking detector for neutron and proton imaging spectroscopy Ryan, James M. and Macri, John R. and McConnell, Mark L. and Miller, Richard S., AIP Conference Proceedings, 576, 571-574 (2001), DOI:http://dx.doi.org/10.1063/1.1395374

This Conference Proceeding is brought to you for free and open access by the Institute for the Study of Earth, Oceans, and Space (EOS) at University of New Hampshire Scholars' Repository. It has been accepted for inclusion in Space Science Center by an authorized administrator of University of New Hampshire Scholars' Repository. For more information, please contact Scholarly.Communication@unh.edu. 


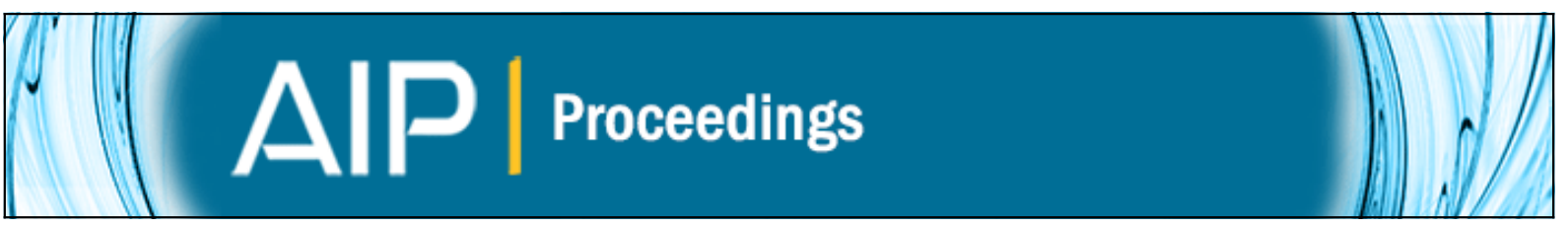

\section{SONTRAC - a scintillating plastic fiber tracking detector for neutron and proton imaging spectroscopy}

James M. Ryan, John R. Macri, Mark L. McConnell, and Richard S. Miller

Citation: AIP Conference Proceedings 576, 571 (2001); doi: 10.1063/1.1395374

View online: http://dx.doi.org/10.1063/1.1395374

View Table of Contents: http://scitation.aip.org/content/aip/proceeding/aipcp/576?ver=pdfcov

Published by the AIP Publishing

\section{Articles you may be interested in}

Monte Carlo study of the energy and angular dependence of the response of plastic scintillation detectors in photon beams

Med. Phys. 37, 5279 (2010); 10.1118/1.3488904

Simulation of the precision limits of plastic scintillation detectors using optimal component selection Med. Phys. 37, 412 (2010); 10.1118/1.3276734

A silicon photomultiplier readout for time of flight neutron spectroscopy with $\mathrm{Y}$-ray detectors

Rev. Sci. Instrum. 80, 095108 (2009); 10.1063/1.3212677

SONTRAC—A low background, large area solar neutron spectrometer

AIP Conf. Proc. 450, 592 (1998); 10.1063/1.56963

Development of FOND: A scintillating fiber-optic neutron detector

AIP Conf. Proc. 450, 555 (1998); 10.1063/1.56960 


\title{
SONTRAC - A Scintillating Plastic Fiber Tracking Detector For Neutron And Proton Imaging Spectroscopy
}

\author{
James M. Ryan, John R. Macri, Mark L. McConnell, Richard S. Miller \\ Space Science Center, Institute for the Study of Earth, Oceans, and Space \\ University of New Hampshire, Durham, NH 03824
}

\begin{abstract}
SONTRAC (SOlar Neutron TRACking imager and spectrometer) is a conceptual instrument intended to measure the energy and incident direction of $20-150 \mathrm{MeV}$ neutrons produced in solar flares. The intense neutron background in a low-Earth orbit requires that imaging techniques be employed to maximize an instrument's signal-tonoise ratio. The instrument is comprised of mutually perpendicular, alternating layers of parallel, scintillating, plastic fibers that are viewed by optoelectronic devices. Two stereoscopic views of recoil proton tracks are necessary to determine the incident neutron's direction and energy. The instrument can also be used as a powerful energetic proton imager. Data from a fully functional 3-d prototype are presented. Early results indicate that the instrument's neutron energy resolution is approximately $10 \%$ with the neutron incident direction determined to within a few degrees.
\end{abstract}

\section{INTRODUCTION}

We are developing an instrument capable of simultaneously performing neutron/proton imaging and spectroscopy. Originally conceived and developed for space-based astrophysics investigations, the SONTRAC (Solar Neutron TRACking telescope) instrument concept has other applications ranging from medical therapy to environmental radiation monitoring. In the SONTRAC concept a bundle of tightly-packed scintillating fibers tracks recoil protons from neutron scattering. The detection and measurement of double scatter events within the fiber bundle permit a determination of the incident energy and direction of each neutron in the $20-250 \mathrm{MeV}$ energy range. Using a scintillating fiber detector built up from orthogonal layers of fiber planes, a full 3dimensional image of recoil proton tracks can be recorded and reconstructed. This technique provides excellent energy and angular resolution in a compact and efficient package for neutron measurements and represents the next generation of space-based neutron telescopes and spectrometers. We have successfully demonstrated the performance of a small 3dimensional SONTRAC prototype, describe that instrument here, and present preliminary performance characteristics.

\section{MOTIVATION}

Besides solar flare neutrons, the technology that will be developed for SONTRAC will benefit any application where directional sensitivity to fast neutrons and/or neutron spectroscopy is important The SONTRAC instrument concept provides a number of unique capabilities for the detection and measurement of neutrons, including:

- Simultaneous determination of energy and direction of each incident neutron

- Angular resolution varying from $<20^{\circ}$ at 20 $\mathrm{MeV}$ to $\sim 1^{\circ}$ at $250 \mathrm{MeV}$

- Energy resolution better than $10 \%$ over the energy range of the telescope

- Ability to distinguish minimum ionizing and highly ionizing particles

- Significant background rejection capabilities

- Portability, large effective area and compactness 


\section{Technical Status Summary}

We summarize the current SONTRAC development status as follows:

- We have an operational $5 \times 5 \times 5 \mathrm{~cm}$ threedimensional science model detector

- There is sufficient signal to detect and track minimum ionizing particles.

- Event-by-event detection and measurement of track length (energy) track direction and particle type has been achieved.

- We have developed track recognition algorithms and used them to analyze proton beam data for characterization of energy and angular resolution capabilities up to $67.5 \mathrm{MeV}$.

\section{The SONTRAC Concept}

The SONTRAC detector can unambiguously reproduce the energy and direction of each incident neutron. The approach is based on the non-relativistic elastic double scatter of neutrons off ambient hydrogen. SONTRAC is based on an earlier concept investigated at Case Western Reserve University (Frye et al. 1985, 1987; Pendleton et al. 1988) and developed, to the level of a small prototype, with NASA Supporting Research \& Technology (SR\&T) funds by the University of New Hampshire.

\section{Basic Principles}

The double-scatter of a non-relativistic neutron in a solid block of plastic scintillator is illustrated in Figure 1 (top). Neutrons interact in plastic scintillator either by elastically scattering from hydrogen $(n-p)$ or by interacting with carbon (n-C). The n-p events are the most useful. For non-relativistic scattering

$$
\sin ^{2} \phi_{n^{\prime}}=\cos ^{2} \phi_{p^{\prime}}=\frac{E_{p^{\prime}}}{E_{n^{\prime}}+E_{p^{\prime}}}=\frac{E_{p^{\prime}}}{E_{n}}
$$

where, $E_{n}$ is the incident neutron energy; $E_{n}$ and $E_{p}$, are the scattered neutron and proton energies, respectively; $\phi_{n}$, and $\phi_{p}$, are the neutron and proton scatter angles, respectively. The kinematics of nonrelativistic elastic scattering implies that the scattered neutron and proton momenta are mutually orthogonal. Relativistic corrections at higher energies are necessary.


FIGURE 1. Schematic of non-relativistic double scatter neutron event in a block of plastic scintillator.

If the incident direction of a given neutron is known, then the measurement of the energy and direction of a recoil proton in a single scattering is sufficient to determine the incident neutron energy. In particular, if the incident direction is known, then $E_{n}$. is determined and the neutron energy is,

$$
E_{n}=\frac{E_{p^{\prime}}}{\cos ^{2} \phi_{p^{\prime}}}
$$

An unambiguous approach, however, is provided by double-scatter events (Figure 1, bottom). If both recoil protons in a double scatter event can be measured, then the energy and incident direction of the neutron are uniquely determined. A system that can measure the parameters of both recoil proton tracks in three dimensions therefore provides the information that is necessary and sufficient to unambiguously determine the incident neutron energy and direction. The angular and energy resolutions depend on the ability to measure the energy and direction of the recoil protons. Because there are no formal restrictions on instrumental field-of-view double scatter events can be used to measure the neutron intensity from an extended sources without significant loss of sensitivity. Double scatter events are also 
preferred, because they allow for a more complete separation of the source signal from the background.

\section{Scintillating Fiber Detector}

We use orthogonal plastic scintillator fiber bundles for measuring neutrons in the 20 to $250 \mathrm{MeV}$ range by recording the image of the recoil proton tracks induced by neutron interactions. The scintillating fibers serve as the source of ambient proton scattering centers, and as light pipes for image readout. The basic instrument consists of a closely packed bundle of square cross section, scintillating fibers. A fiber pitch of $300 \mu \mathrm{m}$ (250 $\mu \mathrm{m}$ active fiber size) was selected so that a 10 $\mathrm{MeV}$ proton traverses several fibers before stopping. The fibers are arranged in stacked planes with the fibers in each plane orthogonal to those in the planes above and below. This alternating orientation of fiber planes provides a stereoscopic view of recoil proton tracks and allows the reconstruction of these tracks in three dimensions. These ionizing tracks in turn provide the information necessary to determine the arrival direction and energy of incident neutrons.

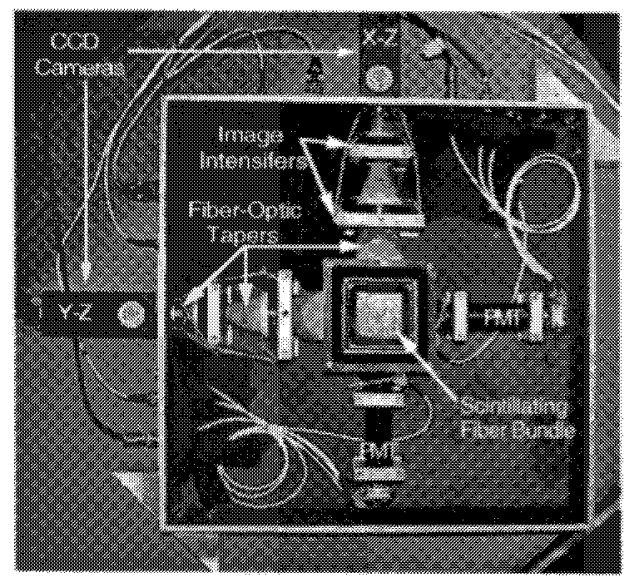

FIGURE 2. SONTRAC SM assembly.

The fibers in each dimension are coupled to imaging electronics (image-intensified CCD cameras, II-CCD). The intensity of each fiber's scintillation light combined with the location of the fiber bundle within the bundle provides the information for reconstruction. As expected, the ionization track length provides a sensitive measure of the recoil proton energy. The Bragg peak, corresponding to the stronger ionization near the end of a proton track, identifies the particle direction.

Early designs of this detector concept were studied with Monte Carlo simulations (Frye et al., 1985, 1987; Pendleton et al., 1988). The concept suffered at the time from a lack of sufficiently mature technology and existed in simulations only. Applicable technologies (small fibers, optoelectronics) have since become commercially available.
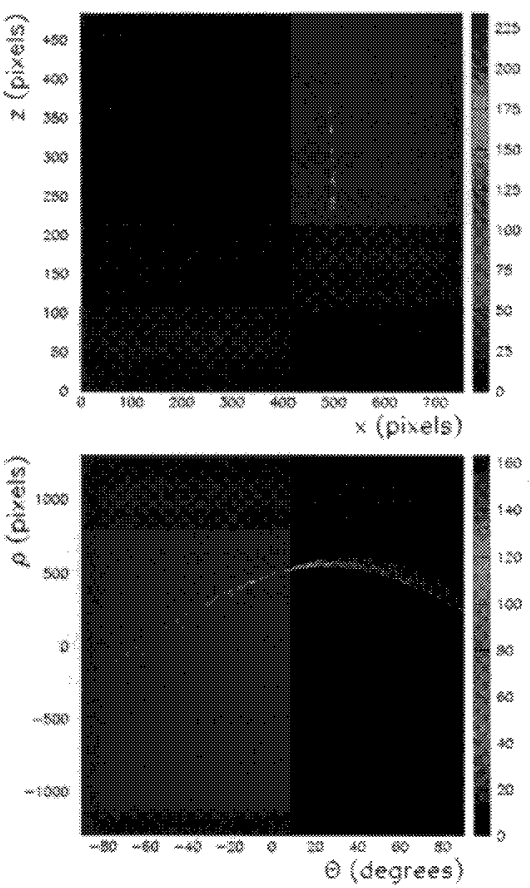

Figure 3. (top) Calibration image showing a normal incidence proton. (bottom) Hough Transform of image.

\section{SONTRAC Science Model}

We have assembled and tested a three-dimensional science model (SM), developed track recognition and data analysis tools, and used these to begin characterizing the performance parameters important for space- and ground-based applications.

The SONTRAC science model is a true 3dimensional version of the SONTRAC instrument concept. The SM fiber bundle is a $5 \times 5 \times 5 \mathrm{~cm}$ scintillating plastic fiber tracking detector with appropriately sized optoelectronic readout components. Figure 2 is a photograph of the assembled SONTRAC SM. The plastic scintillating fiber detector bundle (center) as well as the light guides, fiber optic tapers, image intensifiers, and associated power supplies are contained within a single light-tight enclosure (cover removed in photo). The CCD cameras are mounted externally. The physical size of the optoelectronics required for readout, relative to the active detector area (fiber bundle) is illustrated in the photo. Much of the volume, mass, and cost of the assembly is associated 
with the fiber optic tapers and photomultiplier tubes (PMT). Future designs will eliminate the PMTs and will have alternative readout schemes to replace the IICCD chains.

The SM assembly can be rotated through a $\pm 45^{\circ}$ range of incident angles for exposure to calibrated beams. External equipment, not shown, includes NIM electronics and a CAMAC ADC for processing the

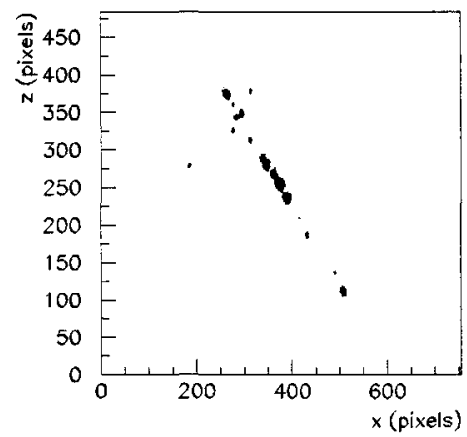

PMT signals and performing the trigger logic, and the computer for control and data acquisition. The SM is self-triggered; a 2-fold coincidence of the PMTs forms the trigger for image acquisition. The $5 \mathrm{~cm}$ size of the SM fiber bundle permits measurements to be made from $10-70 \mathrm{MeV}$, a range starting below the nominal threshold up to energies where neutron double scatter events are no longer contained.

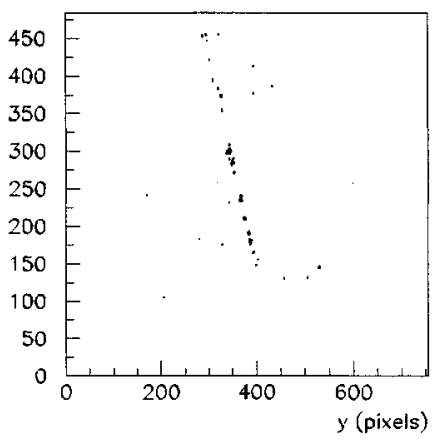

FIGURE 4. Raw CCD images showing orthogonal views of a cosmic ray muon track detected with SONTRAC science model.

\section{Preliminary Calibration Results}

Proton data was acquired with the SM at Crocker Nuclear Laboratory (U.C. Davis) and used to develop image-processing software for event analysis. The Hough Transform (HT) (Duda, et al. 1972; Sklansky 1978) is an effective technique for track reconstruction while simultaneously providing a large degree of data compression. Although other analysis techniques have been applied to the SM images (e.g. chi-square line fit) the HT is faster, and in this application more accurate. The HT also provides information useful for determining event quality. Image reconstruction using the HT effectively compresses the data from roughly 1 megabyte per image to a tuple of a few bytes composed of data such as $\theta, r$, starting points, length, and intensity. A data example (single projection of incident proton) from the SM is shown in Figure 3; Figure 4 shows two orthogonal projections of an incident muon event.

Initial results from the proton calibration effort yield a $1 \sigma$ angular resolution of $1.5^{\circ}$. Similar results were obtained for data taken $\sim 17^{\circ}$ off-axis. Taking track length to be proportional to energy gives an energy resolution in one projection of $\sim 7.5 \%(1 \sigma)$ at $67.5 \mathrm{MeV}$. Calibration of the instrument response to incident protons (and neutrons) at various energies is planned in the near future.

\section{ACKNOWLEDGMENTS}

We have built a fully functional 3-dimensional SONTRAC prototype and performed initial calibrations to determine instrument response. The excellent results from these efforts suggest that larger, more sensitive detectors based on this instrument concept are possible, with unique and useful application to a variety of science disciplines including medical imaging and nuclear physics research.

\section{REFERENCES}

1. Frye, G.M., et al., 20th ICRC, 4, 392, 1987.

2. Frye, G.M., et al., 19th ICRC, 5, 498, 1985

3. Pendleton et al., Workshop on Scint. Fiber Devel., Fermilab, 1093, 1988

4. Duda, Richard O. and Hart, Peter E., Commun. Ass. Comput. Mach., vol. 15, pp 11-15, Jan 1972.

5. Sklansky, Jack, On the Hough Technique for Curve Detection, IEEE Transactions on Computers, vol. C-27, No. 10 , Oct. 1978. 